\title{
EFEITO DO TIPO DE SUBSTRATO E DA PRESENCA DE ADUBAC̃̃o SUPLEMENTAR SOBRE O CRESCIMENTO VEGETATIVO, NUTRIÇÃO MINERAL, CUSTO DE PRODUÇÃO E INTENSIDADE DE CERCOSPORIOSE EM MUDAS DE CAFEEIRO FORMADAS EM TUBETES
}

\author{
Effects of substrate type and supplementary fertilization on vegetative growth, mineral \\ nutrition, production costs, and brown eye spot of coffee seedlings in stiff plastic containers
}

\author{
Adélia Aziz Alexandre Pozza' ${ }^{1}$ Paulo Tácito Gontijo Guimarães ${ }^{2}$ Edson Ampélio Pozza ${ }^{3}$, \\ Janice Guedes de Carvalho ${ }^{4}$, Marcelo Montanari ${ }^{5}$, Renato Ferreira de Souza ${ }^{6}$
}

\begin{abstract}
RESUMO
A utilização de tubetes para produzir mudas de cafeeiro (Coffea arabica L.) tornou-se uma realidade. Objetivando avaliar o uso de substratos adubados ou não com fertilizante de liberação gradual e seus efeitos na nutrição, na formação, na cercosporiose e nos custos de produção de mudas de cafeeiro em tubetes, conduziu-se um experimento em viveiro, em esquema fatorial (4x2) com quatro substratos combinados com duas adubações, tendo quatro repetições. As parcelas foram constituídas por 16 tubetes de $120 \mathrm{~mL}$, considerando-se como plantas úteis as seis centrais. Os tratamentos consistiram dos substratos $\mathrm{S}_{1}=$ Substrato comercial I, $\mathrm{S}_{2}=$ Substrato comercial II, $\mathrm{S}_{3}$ $=$ Substrato de eucalipto e $\mathrm{S}_{4}=80 \%$ esterco de curral $+20 \%$ terra de subsolo, todos com e sem adubação com fertilizante de liberação gradual. No geral, os melhores substratos para formação de mudas de cafeeiro em tubetes foram os substratos não comerciais ou orgânicos, independentemente de receberem ou não adubação suplementar. Observou-se redução da cercosporiose com a nutrição mineral, principalmente com o cálcio. Com a utilização de todos os substratos houve ganho econômico em relação ao custo por milheiro de mudas. A maior economia foi obtida com o substrato $S_{4}$, seguida do substrato $S_{3}$, com melhor relação custo/benefício.
\end{abstract}

Termos para indexação: Nutrição e doença, cafeicultura, Cercospora coffeicola, fertilização, produção de mudas, substrato.

\begin{abstract}
The utilization of stiff plastic containers for producing coffee seedlings (Coffea arabica L.) is a reality. Aiming to evaluate the effects of substrate type enriched or not with slow-release fertilizer on vegetative growth, mineral nutrition, production cost and, brown eye spot of coffee seedlings, an experiment was conducted in the plant nursery. The experimental design was a factorial scheme with four substrates in the presence and absence of supplementary fertilization, with four replicates. The plots were made of 16 containers of $120 \mathrm{ml}$, using six central plants for evaluation. The treatments consisted of $\mathrm{S}_{1}=$ Commercial Substrate $\mathrm{I}, \mathrm{S}_{2}=$ Commercial Substrate II, $S_{3}=$ Eucalyptus Substrate and $S_{4}=80 \%$ bovine manure $+20 \%$ subsoil material with and without fertilization. In general, the best substrates for formation of coffee seedlings were not commercial substrates or organic, regardless of fertilization. The brown eye spot was reduced with balanced nutrition, mainly with calcium. All substrates provide economic return. The largest economy was found in substrate $\mathrm{S}_{4}$, followed by $\mathrm{S}_{3}$, showing the highest coast/benefit rate.
\end{abstract}

Index terms: Nutrition and disease, culture coffee, Cercospora coffeicola, fertilization, seedlings production, substrate.

(Recebido em 8 de setembro de 2005 e aprovado em 6 de março de 2006)

\section{INTRODUÇÃO}

A forma usual de produção de mudas de cafeeiro tem sido por meio da utilização de sacos plásticos e do substrato constituído por $70 \%$ de terra de subsolo e $30 \%$ de esterco de curral, adubado com fertilizantes químicos. Uma alternativa é utilizar recipientes de menor tamanho, a exemplo dos tubetes de plástico rígido (MELO, 1999). Tubetes de plástico apresentam algumas vantagens quando comparados ao sistema tradicional de formação de mudas de cafeeiro, tais como facilidade de manuseio e transporte das mudas, redução da área necessária para o viveiro e menor volume de substrato para encher os tubetes. Nestes recipientes, no entanto, há necessidade de utilizar substratos com características físico-químicas adequadas e com quantidades suficientes de elementos essenciais para o crescimento e desenvolvimento das mudas. Segundo Keever et al. (1985) e Keever \& Cobb (1987), a fertilidade do substrato influi diretamente no desenvolvimento de

\footnotetext{
1Pós-doutoranda/FAPEMIG-Departamento de Ciência do Solo - Universidade Federal de Lavras/UFLA-Cx. P. 3037-37.200-000-Lavras, MG- alana@ufla.br ${ }^{2}$ Pesquisador da EPAMIG/CTSM - Campus da Universidade Federal de Lavras/UFLA - Cx. P. 176 - 37.200-000 - Lavras, MG - paulotgg@ufla.br ${ }^{3}$ Professor Associado do Departamento de Fitopatologia - Universidade Federal de Lavras/UFLA - Cx. P. 3037 - $37.200-000$ - Lavras, MG eapozza@ufla.br

4Professora Titular do Departamento de Ciência do Solo - Universidade Federal de Lavras/UFLA - Cx. P. 3037-37.200-000 - Lavras, MG - janicegc@ufla.br ${ }^{5}$ Engenheiro Agrônomo - Departamento de Ciência do Solo - Universidade Federal de Lavras/UFLA - Cx. P. 3037 - $37.200-000$ - Lavras, MG montanari@ufla.br

${ }^{6}$ Assistente de Pesquisa PRODOC/CAPES - Departamento de Ciência do Solo - Universidade Federal de Lavras/UFLA - Cx. P. 3037 - 37.200-000 Lavras, MG - souzarf@gmail.com
} 
plantas em recipientes. A utilização de fertilizantes de liberação gradual na adubação de substratos para produção de mudas de cafeeiro em tubetes é recente, sendo escassos os trabalhos sobre o assunto disponíveis na literatura (MELO et al., 2001). O custo de produção em tubetes é mais elevado que o custo do sistema tradicional de produção de mudas. Esta diferença é devida ao maior nível tecnológico empregado, à utilização de insumos mais caros, substrato e fertilizante de liberação lenta, e ao maior investimento inicial, o qual é amortizado ao longo dos anos (GUIMARÃES et al., 1998). Portanto, busca-se encontrar um substrato que seja uniforme em sua composição, rico em nutrientes, com elevada capacidade de retenção de água e troca catiônica, isento de pragas, patógenos e sementes de plantas daninhas e viável economicamente.

Nos viveiros, a cercosporiose ou mancha-de-olhopardo (Cercospora coffeicola Berk. \& M.A. Curtis) constitui uma das principais doenças, responsável por desfolha e raquitismo. A intensidade pode agravar-se em decorrência do excesso de irrigação ou deficiência hídrica e da adubação insuficiente e desequilibrada (FERNANDEZBORRERO et al., 1966). Os nutrientes minerais exercem funções específicas no metabolismo vegetal; desse modo, afetam seu crescimento e sua produção. Além disso, a nutrição mineral apresenta envolvimento indireto no metabolismo vegetal, influenciando alguns fatores que podem aumentar ou reduzir a resistência das plantas a patógenos, tais como morfologia (forma de crescimento), anatomia (paredes das células da epiderme mais grossas, lignificadas ou silificadas) e composição química (síntese de compostos tóxicos) (MARSCHNER, 1995).

De acordo com o exposto, pretendeu-se neste trabalho avaliar o efeito de diferentes composições de substratos, adubados ou não com fertilizante de liberação gradual, na nutrição, na cercosporiose, na produção e nos custos de produção de mudas de cafeeiro (Coffea arabica L.) em tubetes.

\section{MATERIAL E MÉTODOS}

$\mathrm{O}$ experimento foi instalado em delineamento experimental inteiramente casualizado, com quatro substratos combinados com dois níveis de adubação e quatro repetições. As parcelas foram constituídas por 16 tubetes de $120 \mathrm{~cm}^{3}$, considerando-se como área útil os seis centrais, dispostos, nas células da bandeja, conforme apresentado na Figura 1.

Os tratamentos foram os seguintes: S1 - Substrato comercial I ("Plantimax"), S2 - Substrato comercial II ("Mecplant"), S3 - Substrato usado para a produção de mudas de eucalipto (30\% de casca de arroz carbonizada, $10 \%$ de terra de subsolo, $40 \%$ de esterco de curral e $20 \%$ de vermiculita) e S4 - Substrato constituído de $80 \%$ esterco de curral $+20 \%$ terra de subsolo. Testou-se também o efeito da adubação com fertilizante de liberação gradual na dose de $10 \mathrm{~kg}$ da formulação 15-10-10 + micronutrientes, granulado e resinado ("osmocote") $/ \mathrm{m}^{3}$ de substrato ou $1 \mathrm{~g}$ por tubete, os quais foram incorporados ao substrato.

A composição dos substratos não comerciais, nas porcentagens em volume, foi realizada utilizando-se um recipiente graduado, sendo os ingredientes colocados em saco plástico com capacidade para 60 litros e agitados em movimentos regulares a fim de homogeneizar a mistura. $\mathrm{O}$ fertilizante foi adicionado aos tratamentos e homogeneizado pelo mesmo processo, por cerca de 2 minutos. Após o enchimento dos recipientes e umedecimento do substrato por irrigação, realizou-se o transplantio utilizando-se plântulas no estádio de "palito de fósforo", obtidas em germinador de areia. Foram realizadas quatro avaliações quinzenais da incidência e da

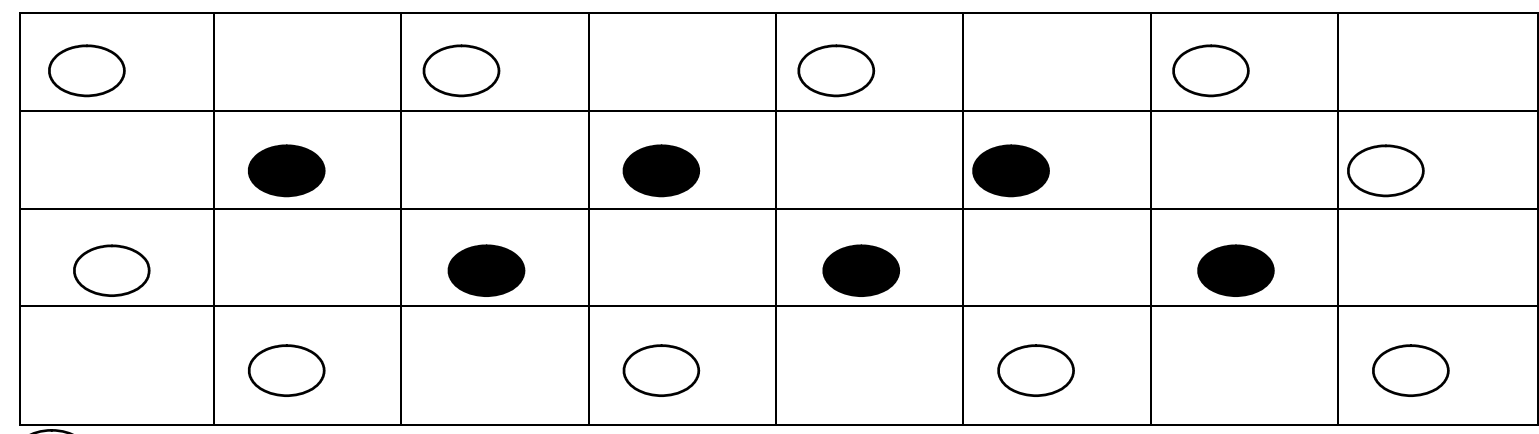

Tubetes com plantas da bordadura.

Tubete com plantas da parcela útil.

FIGURA 1 - Disposição das plantas na parcela útil dentro da bandeja de tubetes.

Ciênc. agrotec., Lavras, v. 31, n. 3, p. 685-692, maio/jun., 2007 
severidade do ataque da cercosporiose. A incidência foi avaliada pela porcentagem de plantas com cercosporiose na parcela e pelo número de folhas lesionadas por planta. A severidade pela contagem do número de lesões por folha e pelo total de lesões por planta. Estes dados foram transformados em área abaixo da curva de progresso da doença (AACPD), de acordo com a equação proposta por Campbell \& Madden (1990). Realizou-se análise de variância em esquema fatorial 4 X 2. As variáveis significativas pelo teste $\mathrm{F}$ foram submetidas ao teste de Scott-Knot, a 5\% de probabilidade. Na avaliação final realizada quando as plantas apresentaram seis pares de folhas, considerou-se altura de planta, número total de internódios, número total de folhas, e pesos da matéria seca do sistema radicular, da parte aérea e total. A análise nutricional realizada para determinar os teores de N, P, K, Ca, Mg, S, B, Cu, Zn, Fe e $\mathrm{Mn}$ de cada tratamento, foi realizada segundo a metodologia descrita por Malavolta et al. (1997).

Realizou-se um estudo dos custos de produção das mudas em tubetes para cada substrato. Na avaliação dos recursos fixos utilizou-se a depreciação apropriada, pelo método linear. Os recursos analisados no processo produtivo de mudas de café foram: materiais para construção do viveiro, materiais para construção dos germinadores, irrigação, taxa de registro do viveiro e outros custos fixos gerais. Quanto aos custos variáveis, consideraram-se as despesas com mão-de-obra permanente e temporária, insumos (substrato, fertilizantes, fungicidas, inseticidas, sementes, etc.), assessoria e despesas gerais. Para efeito de análise do custo alternativo dos recursos produtivos alocados no viveiro de mudas considerou-se a taxa de juros de 6\% a.a. (BIOEX, 1999).

\section{RESULTADOS E DISCUSSÃO}

$\mathrm{Na}$ ausência de adubação, os maiores valores de altura das mudas, número de internódios e peso das raízes secas, foram obtidos no substrato S3. Ao adubar-se o substrato, o número de internódios não diferiu entre os substratos e o $\mathrm{S} 1$ foi inferior aos demais quanto à altura, peso da parte aérea seca, das raízes secas e da matéria seca total, igualando-se ao S4 quanto ao total de folhas das plantas (Tabela 1).

De modo geral, na ausência de adubação, as menores áreas abaixo da curva de progresso (AACP) do número de plantas com cercosporiose (PD), do número de folhas lesionadas (NFL), do total de lesões (TL) e do número de lesões por folha (NLF), foram obtidas no substrato S3, seguido pelo substrato S4, e as maiores AACP, nos substratos S1 e S2 (Tabela 2). Os tratamentos com adubação não diferiram significativamente entre si. A menor incidência de cercosporiose observada nos substratos S3 e S4, provavelmente, deveu-se a um melhor equilíbrio e disponibilidade de nutrientes nestes substratos do que nos substratos S1 e S2, pois plantas mais bem nutridas apresentam menor incidência de cercosporiose (FERNANDEZ-BORRERO et al., 1966). Nos tratamentos com fertilizante houve fornecimento adequado de nutrientes, por isso os tratamentos não diferiram entre si.

TABELA 1 - Médias dos valores de altura de plantas (Altura), número total de internódios por planta (Internódios), número total de folhas por planta (Total folhas), peso da parte aérea seca (Parte aérea), peso das raízes secas (Raiz) e matéria total seca (MST) de mudas de cafeeiros formadas em diferentes substratos (Trat), com e sem adubação.

\begin{tabular}{|c|c|c|c|c|c|c|c|c|c|c|c|c|}
\hline \multirow[t]{3}{*}{ Trat. } & \multicolumn{2}{|c|}{ Altura } & \multicolumn{2}{|c|}{ Internódios } & \multicolumn{2}{|c|}{ Total folhas } & \multicolumn{2}{|c|}{ Parte aérea } & \multicolumn{2}{|c|}{ Raiz } & \multicolumn{2}{|c|}{ MST } \\
\hline & \multicolumn{12}{|c|}{ Adubação com fertilizante de liberação gradual } \\
\hline & Com & Sem & Com & Sem & Com & Sem & Com & Sem & Com & Sem & Com & Sem \\
\hline S1 & $12,2 b$ & $6,7 \mathrm{c}$ & $6,5 \mathrm{a}$ & $4,9 \mathrm{c}$ & $12,1 \mathrm{~b}$ & $9,4 \mathrm{~b}$ & $2,9 \mathrm{c}$ & $1,7 \mathrm{c}$ & $1,0 \mathrm{~b}$ & $1,3 b$ & $3,9 b$ & $2,9 b$ \\
\hline $\mathrm{S} 2$ & $15,2 \mathrm{a}$ & $6,9 \mathrm{c}$ & $6,7 \mathrm{a}$ & $4,7 \mathrm{c}$ & $13,4 a$ & $6,8 \mathrm{c}$ & $4,4 b$ & $1,3 \mathrm{c}$ & $1,4 \mathrm{a}$ & $1,3 b$ & $5,8 \mathrm{a}$ & $2,5 b$ \\
\hline $\mathrm{S} 3$ & $15,8 \mathrm{a}$ & $14,4 \mathrm{a}$ & $6,7 \mathrm{a}$ & $6,5 a$ & $13,4 \mathrm{a}$ & $12,6 a$ & $5,1 \mathrm{a}$ & $4,4 \mathrm{~b}$ & $1,3 \mathrm{a}$ & $1,5 \mathrm{a}$ & $6,4 \mathrm{a}$ & $5,9 \mathrm{a}$ \\
\hline S4 & $15,5 \mathrm{a}$ & $10,8 b$ & $6,4 a$ & $5,8 \mathrm{~b}$ & $12,5 b$ & $11,9 a$ & $4,8 \mathrm{a}$ & $5,4 \mathrm{a}$ & $1,6 a$ & $1,1 b$ & $6,4 \mathrm{a}$ & $6,5 \mathrm{a}$ \\
\hline $\mathrm{CV} \%$ & \multicolumn{2}{|c|}{5,55} & \multicolumn{2}{|c|}{4,82} & \multicolumn{2}{|c|}{6,73} & \multicolumn{2}{|c|}{8,68} & \multicolumn{2}{|c|}{11,68} & \multicolumn{2}{|c|}{7,93} \\
\hline
\end{tabular}

Médias seguidas de mesma letra na coluna não diferem estatisticamente entre si, pelo teste de Tukey a 5\% de probabilidade. 
TABELA 2 - Médias do número de plantas doentes por parcela de seis plantas (PD), número de folhas lesionadas por planta (NFL), total de lesões por planta (TL) e número de lesões de C. coffeicola por folha (NLF), expressos como área abaixo da curva de progresso da cercosporiose $\left(\mathrm{mm}^{2}\right)$, em mudas de cafeeiros formadas em diferentes substratos (Trat.), com e sem adubação.

\begin{tabular}{|c|c|c|c|c|c|c|c|c|}
\hline \multirow[t]{3}{*}{ Trat. } & \multicolumn{2}{|c|}{ PD } & \multicolumn{2}{|c|}{ NFL } & \multicolumn{2}{|c|}{ TL } & \multicolumn{2}{|c|}{ NLF } \\
\hline & \multicolumn{8}{|c|}{ Adubação com fertilizante de liberação gradual } \\
\hline & Com & Sem & Com & Sem & Com & Sem & Com & Sem \\
\hline S1 & $56 a$ & $1208 b$ & $87 \mathrm{a}$ & $2061 b$ & $156 a$ & $2095 b$ & $383 a$ & $429 a$ \\
\hline $\mathrm{S} 2$ & $317 \mathrm{a}$ & $2209 a$ & $394 a$ & $3069 a$ & $473 a$ & $5164 a$ & $398 a$ & $470 \mathrm{a}$ \\
\hline $\mathrm{S} 3$ & $52 \mathrm{a}$ & $279 d$ & $52 \mathrm{a}$ & $113 d$ & $52 \mathrm{a}$ & $120 \mathrm{c}$ & $375 a$ & $357 b$ \\
\hline $\mathrm{S} 4$ & $110 \mathrm{a}$ & 901c & $149 a$ & $1136 \mathrm{c}$ & $379 a$ & $1495 b$ & $383 a$ & $433 a$ \\
\hline $\mathrm{CV} \%$ & & & & & & & & \\
\hline
\end{tabular}

Médias seguidas de mesma letra na coluna, não diferem estatisticamente entre si, pelo teste de Tukey a 5\% de probabilidade.

As plantas crescidas no substrato S1 com fertilizante, apresentaram maiores teores de $\mathrm{N}$ na parte aérea (Tabela 3), provavelmente devido ao efeito de concentração ou efeito Steenbjerg, fenômeno que resulta da combinação de concentração do elemento e redução da matéria seca da planta (JONES JUNIOR et al., 1991), pois neste substrato encontram-se as menores mudas e os menores pesos de matéria seca (Tabela 1). Como o $\mathrm{N}$ é bastante móvel na planta, estando disponível, ele é o nutriente que reflete primeiro esse efeito de concentração. Nas plantas do substrato S3 não adubado, e em seguida nas do substrato $\mathrm{S} 4$, ocorreram menores intensidades de cercosporiose, provavelmente devido aos maiores teores de $\mathrm{N}$ nesses substratos. O N é essencial para produção de aminoácidos, proteínas, hormônios de crescimento e fitoalexinas (HUBER, 1980). A ação das fitoalexinas sobre fungos dá-se, sobretudo pela inibição da elongação do tubo germinativo, sendo o ápice das hifas sensível a fitoalexinas, devido à inibição das enzimas envolvidas na síntese da parede celular, que resulta no intumescimento e ruptura das hifas (RESENDE \& CARVALHO, 2002). Da mesma forma, os menores teores de $\mathrm{N}$ (14,2 e 14,8 $\mathrm{g} / \mathrm{kg}$ respectivamente) foram obtidos nos substratos S2 e S1 (Tabela 3), os quais tiveram maiores intensidades de doença (Tabela 2). Pozza et al. (2001), em experimento com doses crescentes de $\mathrm{N}$ e de $\mathrm{K}$ em mudas de cafeeiro com 6,5 meses de idade em solução nutritiva, também ressaltaram redução na severidade da cercosporiose em doses maiores de N. Nesse trabalho não se observou diferença estatística nos teores de $\mathrm{K}$ entre os tratamentos.

Os teores de $\mathrm{P}$ na parte aérea foram maiores no substrato S2 com fertilizante e no substrato S4 sem fertilizante (Tabela 3). Entretanto esses teores, variando de 2,1 a $3,4 \mathrm{~g} / \mathrm{kg}$, foram maiores que os considerados adequados para cafeeiros adultos (1,6 a $1,9 \mathrm{~g} / \mathrm{kg})$, por Malavolta et al. (1997), e semelhantes (1,6 a 2,9 g/kg) aos encontrados por Braccini (1995), Pozza et al. (2001) e Rodrigues (1997). Esses autores os consideraram adequados para mudas de cafeeiro com seis a sete meses de idade. $O$ fósforo quando em deficiência resulta em menor síntese protéica, redução dos alcalóides, lignina e glicosídeos (MARSCHNER, 1995) e sua combinação com potássio tem sido considerada importante na redução do ataque da cercosporiose do cafeeiro (ZAMBOLIN \& VENTURA, 1993). Entretanto, segundo os mesmos autores, o aumento do nível de fósforo em certos casos pode aumentar a severidade da doença, como ocorre com a ferrugem da cana. Nenhuma dessas variações foi observada nesse experimento (comparação das Tabelas 1, 2 e 3) .

Os teores de $\mathrm{Ca}$ apresentaram-se mais baixos no substrato S2 sem adubação, quando comparado aos demais. Pozza et al. (2001) encontraram faixa de concentração de Ca entre 9,6 a 13,6 g/kg e consideraram-na não limitante ao crescimento e desenvolvimento de mudas de cafeeiro, entretanto, concentrações iguais ou abaixo do limite inferior permitiram maior severidade da cercosporiose.

Ciênc. agrotec., Lavras, v. 31, n. 3, p. 685-692, maio/jun., 2007 
TABELA 3 - Médias dos teores dos macronutrientes na parte aérea de mudas de cafeeiros produzidas em diferentes substratos (Trat.) com e sem adubação.

\begin{tabular}{|c|c|c|c|c|c|c|c|c|c|c|}
\hline \multirow[t]{3}{*}{ Trat. } & \multicolumn{2}{|c|}{$\mathbf{N}$} & \multicolumn{2}{|c|}{$\mathbf{P}$} & \multicolumn{2}{|c|}{$\mathbf{C a}$} & \multicolumn{2}{|c|}{ Mg } & \multicolumn{2}{|c|}{$\mathbf{S}$} \\
\hline & \multicolumn{10}{|c|}{ Adubação com fertilizante de liberação gradual } \\
\hline & Com & Sem & Com & Sem & Com & Sem & Com & Sem & Com & Sem \\
\hline \multicolumn{11}{|c|}{ 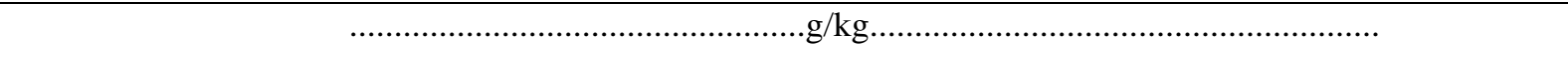 } \\
\hline $\mathrm{S} 1$ & $35,2 \mathrm{a}$ & $14,8 b$ & $2,4 b$ & $2,1 b$ & $9,5 b$ & $10,4 b$ & $4,6 a$ & $4,9 a$ & $1,2 \mathrm{a}$ & $0,8 \mathrm{a}$ \\
\hline $\mathrm{S} 2$ & $23,5 b$ & $14,2 \mathrm{~b}$ & $3,1 \mathrm{a}$ & $3,3 b$ & $8,7 b$ & $8,7 \mathrm{c}$ & $4,7 \mathrm{a}$ & $4,9 a$ & $0,9 b$ & $0,5 b$ \\
\hline $\mathrm{S} 3$ & $27,0 \mathrm{~b}$ & $25,7 \mathrm{a}$ & $2,6 b$ & $2,5 b$ & $10,4 \mathrm{a}$ & $12,0 \mathrm{a}$ & $4,0 \mathrm{~b}$ & $4,7 \mathrm{a}$ & $0,9 b$ & $0,8 \mathrm{a}$ \\
\hline $\mathrm{S} 4$ & $23,7 b$ & $24,9 \mathrm{a}$ & $2,1 b$ & $3,4 a$ & $9,0 \mathrm{~b}$ & $10,1 b$ & $3,8 b$ & $5,1 \mathrm{a}$ & $0,7 \mathrm{c}$ & $0,6 b$ \\
\hline $\mathrm{CV} \%$ & \multicolumn{2}{|c|}{10,56} & \multicolumn{2}{|c|}{11,44} & \multicolumn{2}{|c|}{5,80} & \multicolumn{2}{|c|}{5,87} & \multicolumn{2}{|c|}{13,91} \\
\hline
\end{tabular}

Médias seguidas de mesma letra na coluna não diferem estatisticamente entre si pelo teste de Tukey a 5\% de probabilidade.

Nesse experimento as plantas do substrato com maior intensidade de doença continham em sua parte aérea, teores da ordem de $8,7 \mathrm{~g} / \mathrm{kg}$, o qual pode ter promovido menor crescimento e desenvolvimento das mudas (Tabela 1) e maior intensidade da doença (Tabela 2). De acordo com Agrios (1988), Huber (2002) e Marschner (1995), o cálcio reduz a severidade de várias doenças por constituir a lamela média, presente principalmente na forma de pectatos de cálcio, conferindo resistência à penetração do patógeno. Castaño (1956) comprovou a penetração de C. coffeicola por estômatos e diretamente através da cutícula. Nela, o cálcio assume grande importância, devido a sua função na estabilização de paredes e membranas celulares, onde atua como agente cimentante, aumentando a resistência à penetração de patógenos. Para o cafeeiro, Garcia Júnior (2002) observou redução linear da incidência de cercosporiose com aumento do teor de Ca nas folhas.

Os teores foliares de $\mathrm{Mg}(3,8$ a $5,1 \mathrm{~g} / \mathrm{kg})$ encontraram-se acima da faixa considerada adequada por Braccini (1995) e Rodrigues (1997), a qual situa-se entre 2,7 a $3,8 \mathrm{~g} / \mathrm{kg}$, entretanto, parece que esses valores não afetaram o crescimento das mudas (Tabela 3 ). Os teores de enxofre $(0,5$ a $1,2 \mathrm{~g} / \mathrm{kg})$ ficaram abaixo dos considerados adequados (1,5 a 2,0 g/kg) por Malavolta et al. (1997). Não foram encontradas na literatura citações de sua relação com o cafeeiro, entretanto, o S tem importante papel no metabolismo, é incorporado a aminoácidos, proteínas, enzimas, vitaminas, óleos aromáticos e ferredoxinas
(MARSCHNER, 1995), podendo dessa forma atuar na cercosporiose do cafeeiro.

Independentemente do substrato, as menores áreas abaixo da curva de progresso da cercosporiose, ou seja a menor intensidade da doença e os maiores valores de altura, internódios, total de folhas, parte aérea e matéria total seca foram obtidos quando efetuou-se adubação (Tabela 4).

É interessante observar na parte aérea das plantas dos substratos $\mathrm{S} 2$ e S1, sem adubação, elevados teores de B e Fe, e no S2 elevado teor de Mn, com e sem adubação, indicando ser este, um substrato rico em micronutrientes (Tabela 5). Malavolta (1993) considera teores excessivos do $\mathrm{Mn}$ aqueles maiores que $300 \mathrm{mg} / \mathrm{kg}$. No substrato S2 foram constatados teores de 312,1 e $308,0 \mathrm{mg} / \mathrm{kg}$ (Tabela 5). Alta concentração de Mn pode inibir por competição, a absorção de $\mathrm{Fe}$ e a sua translocação, nutriente responsável pela ativação de enzimas envolvidas na redução do nitrato, no metabolismo de carboidratos e na respiração (HUBER, 1980). Esta competição parece não ter ocorrido nesse experimento, porque os teores de $\mathrm{Fe}$ do $\mathrm{S} 2$ foram normais.

Os teores de Fe variaram de 50,4 a 106,0 mg/kg (Tabela 5). Rodrigues (1997) encontrou concentrações de 70 a $200 \mathrm{mg} / \mathrm{kg}$ em plantas de café com 6,5 meses de idade e considerou-as adequadas. Os baixos valores encontrados nesse experimento provavelmente devem-se a alta porcentagem de matéria orgânica contida nos substratos, a qual complexa esse metal, tornando-o indisponível. 
TABELA 4 - Médias dos valores do número de plantas doentes por parcela (PD), número de folhas lesionadas por planta (NFL), número total de lesões (TL), número de lesões de $C$. coffeicola por folha (NLF) expressas como a área abaixo da curva de progresso da doença, altura média das plantas (altura), total de internódios (Intern.), número total de folhas (Tfolh), peso da parte aérea seca (PA), peso das raízes secas (Raiz) e matéria seca total (MST) das mudas de cafeeiro formadas em tubetes com e sem adubação do substrato.

\begin{tabular}{ccccccccccc}
\hline Adub & PD & NFL & TL & NLF & Altura & Intern. & Tfolh & PA & Raiz & MST \\
\hline Com & $134 \mathrm{~b}$ & $171 \mathrm{~b}$ & $265 \mathrm{~b}$ & $385 \mathrm{~b}$ & $14,7 \mathrm{a}$ & $6,6 \mathrm{a}$ & $12,9 \mathrm{a}$ & $4,3 \mathrm{a}$ & $1,3 \mathrm{~ns}$ & $5,6 \mathrm{a}$ \\
Sem & $1149 \mathrm{a}$ & $1594 \mathrm{a}$ & $2218 \mathrm{a}$ & $422 \mathrm{a}$ & $9,7 \mathrm{~b}$ & $5,5 \mathrm{~b}$ & $10,2 \mathrm{~b}$ & $3,2 \mathrm{~b}$ & $1,3 \mathrm{~ns}$ & $4,5 \mathrm{~b}$ \\
\hline
\end{tabular}

TABELA 5 - Teores dos micronutrientes na parte aérea de mudas de cafeeiro em diferentes substratos (Trat.) com e sem adubação.

\begin{tabular}{|c|c|c|c|c|c|c|c|c|c|c|}
\hline \multirow[t]{3}{*}{ Trat. } & \multicolumn{2}{|c|}{ B } & \multicolumn{2}{|c|}{$\mathbf{C u}$} & \multicolumn{2}{|c|}{$\mathbf{Z n}$} & \multicolumn{2}{|c|}{$\mathrm{Fe}$} & \multicolumn{2}{|c|}{ Mn } \\
\hline & \multicolumn{10}{|c|}{ Adubação com fertilizante de liberação gradual } \\
\hline & Com & Sem & Com & Sem & Com & Sem & Com & Sem & Com & Sem \\
\hline \multicolumn{11}{|c|}{ 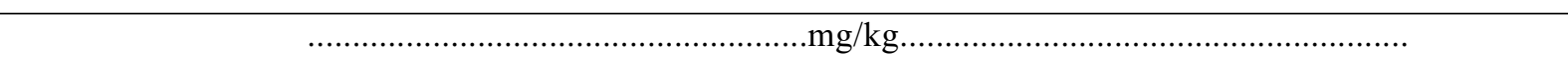 } \\
\hline $\mathrm{S} 1$ & $47,8 \mathrm{a}$ & $92,3 \mathrm{a}$ & $3,7 \mathrm{a}$ & $4,7 b$ & $32,6 \mathrm{a}$ & $50,5 b$ & $55,8 \mathrm{a}$ & $96,5 \mathrm{a}$ & $125,8 \mathrm{~d}$ & $91,8 \mathrm{c}$ \\
\hline $\mathrm{S} 2$ & $64,1 \mathrm{a}$ & $80,7 \mathrm{a}$ & $2,0 \mathrm{a}$ & $4,4 b$ & $27,6 \mathrm{a}$ & $69,6 \mathrm{a}$ & $50,4 \mathrm{a}$ & $106,0 \mathrm{a}$ & $312,1 \mathrm{a}$ & $308,0 \mathrm{a}$ \\
\hline $\mathrm{S} 3$ & $49,5 \mathrm{a}$ & $54,9 \mathrm{~b}$ & $2,9 \mathrm{a}$ & $1,7 \mathrm{c}$ & $25,3 \mathrm{a}$ & $50,9 \mathrm{~b}$ & $51,8 \mathrm{a}$ & $66,7 \mathrm{c}$ & $175,2 \mathrm{c}$ & $101,8 \mathrm{c}$ \\
\hline $\mathrm{S} 4$ & $45,0 \mathrm{a}$ & $61,7 b$ & $2,4 a$ & $7,7 \mathrm{a}$ & $40,6 a$ & $36,3 b$ & $62,0 \mathrm{a}$ & $84,0 \mathrm{~b}$ & $269,5 b$ & $124,8 b$ \\
\hline $\mathrm{CV} \%$ & \multicolumn{2}{|c|}{18,10} & \multicolumn{2}{|c|}{24,81} & \multicolumn{2}{|c|}{34,21} & \multicolumn{2}{|c|}{11,91} & \multicolumn{2}{|c|}{8,07} \\
\hline
\end{tabular}

Médias seguidas de mesma letra na coluna não diferem estatisticamente entre si, pelo teste de Tukey a 5\% de probabilidade.

A participação percentual dos custos fixos e variáveis no custo total dos substratos $\mathrm{S}_{1} \mathrm{~S}_{2}, \mathrm{~S}_{3}$ e $\mathrm{S}_{4}$, constam da Figura 2. O custo total médio por milheiro $(\mathrm{S} 1=$ $\mathrm{R} \$ 97,53, \mathrm{~S} 2=\mathrm{R} \$ 97,53, \mathrm{~S} 3=\mathrm{R} \$ 81,61$ e S4=R $\$ 77,61)$ para todos os substratos, foi inferior ao preço médio de mercado (R\$160,00) do milheiro de mudas. Com a utilização desses substratos, houve maior economia e redução de custos ou lucro econômico, comparado ao preço de mercado para a compra destas mudas de viveiristas, cobrindo todos os recursos aplicados na atividade econômica e proporcionando um ganho para o produtor. Com o substrato $\mathrm{S}_{4}$ o lucro, ou benefício obtido com este substrato, foi $31,89 \%$ maior do que o lucro obtido com o substrato $\mathrm{S}_{1}$ e $\mathrm{S}_{2}$, e $5,10 \%$ maior que o lucro com o substrato $\mathrm{S}_{3}$. Para o substrato $\mathrm{S}_{3}$ o lucro foi $25,48 \%$ maior do que o lucro com o substrato $S_{1}$ e $S_{2}$. Esses maiores lucros foram obtidos devido ao menor custo do substrato utilizado para produção das mudas.

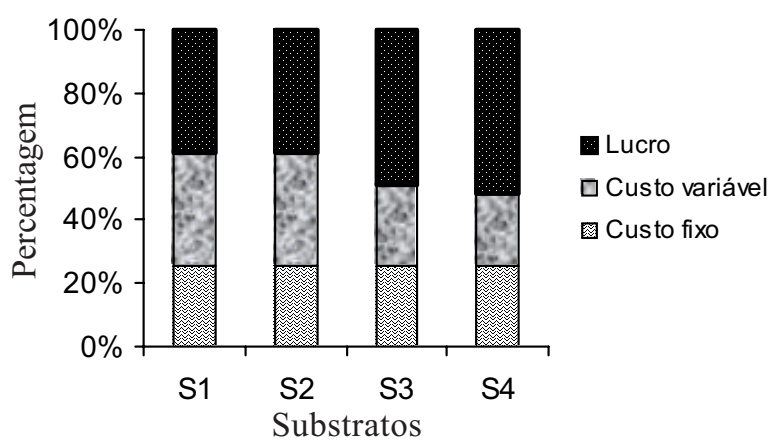

FIGURA 2 - Participação percentual dos custos fixos e variáveis no custo total de produção de mudas de cafeeiro cultivados em diferentes substratos. 


\section{CONCLUSÕES}

No geral, os melhores substratos para formação de mudas de cafeeiro em tubetes foram os substratos não comerciais ou orgânicos (S3 e S4), independentemente de receberem ou não adubação suplementar. Observou-se relação entre a cercosporiose e a nutrição mineral, principalmente com o cálcio. Além disso, os substratos S3 e S4 apresentaram menores custos de produção do que os substratos comerciais S1 e S2 e ganho econômico em relação ao custo por milheiro de mudas.

\section{AGRADECIMENTOS}

Ao Consórcio Brasileiro de Pesquisa e Desenvolvimento do Café - CBP\&D-Café, pelo financiamento do projeto e pela bolsa de estudos da primeira autora e à Empresa de Pesquisa Agropecuária de Minas Gerais EPAMIG/CTSM, pela utilização de sua estrutura para desenvolvimento do projeto.

\section{REFERÊNCIAS BIBLIOGRÁFICAS}

AGRIOS, G. M. Plant pathology. 3. ed. London: Academic, $1988.803 \mathrm{p}$.

BIOEX-Café/CNPq. Como calcular o custo de produção. Lavras: UFLA/CNPq, 1999. 15 p. (Informativo técnico do café, 3).

BRACCINI, M. do C. L. Comportamento de nove populações de café quanto à tolerância ao alumínio em solução nutritiva. 1995. 92 f. Dissertação (Mestrado em Fitotecnia) - Universidade Federal de Viçosa, Viçosa, 1995.

CAMPBELL, C. L.; MADDEN, L. V. Introduction to plant disease epidemiology. New York: J. Wiley \& Sons. 1990. $532 \mathrm{p}$.

CASTAÑO, A. J. J. Mancha de hierro del cafeto. Cenicafe, Chinchiná, v. 82, n. 2, p. 313-327, 1956.

FERNANDEZ-BORRERO, O.; MESTRE, A. M.; DUQUE, S. I. L. Efecto de la fertilización en la incidência de la mancha de hierro(Cercospora coffeicola) en frutos de café. Cenicafe, Chinchiná, v. 17, n. 1, p. 5-16, 1966.

GARCIA JÚNIOR, D. Incidência e severidade da cercosporiose do cafeeiro (Coffea arabica $\mathrm{L}$.) em função das doses de potássio e cálcio em solução nutritiva. 2002.
59 p. Dissertação (Mestrado em Fitopatologia) Universidade Federal de Lavras, Lavras, 2002.

GUIMARÃES, P. T. G.; ANDRADE NETO, A.; BELLINI JUNIOR, O.; ADÃO, W. A.; SILVA, E. M. A produção de mudas de cafeeiros em tubetes. In: TORRES, G.; AMARAL, R.; FRANCO, M.; ANDRADE, A. M. S.; ALVARENGA, L. R.; FAÇANHA, J. B.; CHAVES, C. A. S.; LACERDA, V. L. A. (Eds.). Cafeicultura: tecnologia para produção. Belo Horizonte: EPAMIG, 1998. p. 98-109.

HUBER, D. M. The role of mineral nutrition in defense. In: HORSFAll, J. G.; COWLING, E. B. (Eds.). Plant Pathology: an advanced treatise. New York: Academic, 1980. p. 381406.

HUBER, D. M. Relationship between mineral nutrition of plants and disease incidence. In: WORKSHOP SOBRE RELAÇÃO ENTRE NUTRIÇÃO DE PLANTAS E INCIDÊNCIA DE DOENÇAS, 2002, Piracicaba. Anais... Piracicaba: ESALQ, 2002. CD-ROM.

JONES JUNIOR, J. B.; WOLF, B.; MILLIS, H. A. Plant analysis handbook. Athens: Micro-macro, 1991. 214 p.

KEEVER, G. J.; COBB, G. S. Effects of container volume and fertility rate on growth of two woody ornamentals. Hortscience, Alexandria, v. 22, n. 5, p. 891-893, Oct. 1987.

KEEVER, G. J.; COBB, G. S.; REED, R. B. Effects of container dimension and growth of three woody ornamentals. Hortscience, Alexandria, v. 20, n. 2, p. 276278, Apr. 1985

MALAVOLTA, E. Nutrição mineral e adubação do cafeeiro: colheitas máximas econômicas. São Paulo: Agronômica Ceres, 1993. 210 p.

MALAVOLTA, E.; VITTI, G. C.; OLIVEIRA, S. A. Avaliação do estado nutricional das plantas: princípios e aplicações. 2. ed. rev. e atual. Piracicaba: Potafos, 1997.

MARSCHNER, H. Mineral nutrition of higher plants. 2. ed. New York: Academic, 1995. 889 p.

MELO, B. Estudos sobre produção de mudas de cafeeiro(Coffea arabica L.) em tubetes. 1999. 119 f. Tese (Doutorado em Fitotecnia) - Universidade Federal de Lavras, Lavras, 1999. 
MELO, B.; MENDES, A. N. G.; GUIMARÃES, P. T. G. Doses crescentes de fertilizante de liberação gradual na produção de mudas de cafeeiro (Coffea arabica L.). Bioscience, Uberlândia, v. 17, n. 1, p. 97-113, jun. 2001.

POZZA, A. A. A.; MARTINEZ, H. E. P.; CAIXETA, S. L.; CARDOSO, A. A.; ZAMBOLIM, L.; POZZA, E. A. Influência da nutrição mineral na intensidade da mancha de olho pardo em mudas de cafeeiro. Pesquisa Agropecuária Brasileira, Brasília, v. 36, n. 1, p. 53-60, jan. 2001.

RESENDE, M. L. V. de; CARVALHO, E. M. de. Fisiologia do parasitismo na pós-colheita: mecanismos de defesa do tecido vegetal. In: POZZA, E. A.; OLIVEIRA, F. A. de (Eds.).
In: SIMPÓSIO DE CONTROLE DE DOENÇAS DE PLANTAS - PATOLOGIA PÓS-COLHEITA DE FRUTAS E HORTALIÇAS, 2., 2002, Lavras, MG. Palestras expandidas... Lavras: UFLA/FAEPE, 2002. 182 p.

RODRIGUES, L. A. Crescimento e composição mineral na arte aérea e nas raízes de duas variedades de café em resposta à calagem na subsuperfície do solo. $1997.89 \mathrm{f}$. Dissertação (Mestrado em Fitotecnia) - Universidade Federal de Viçosa, Viçosa, 1997.

ZAMBOLIM, L.; VENTURA, J. A resistência a doenças induzida pela nutrição mineral das plantas. In: LUZ, W. C. Revisão anual de patologia de plantas. Passo Fundo: UPF, 1993. p. 275-309. 